\title{
Uso del Inventario de los Cinco Grandes en una muestra colombiana
}

\section{Use of the Big five Inventory in a Colombian Sample Uso do Inventário dos Cinco Grandes em uma amostra colombiana}

\author{
Elvira Salgado*, Elvia Vargas-Trujillo*, Jana Schmutzler**, Eduardo Wills-Herrera* \\ *Universidad de los Andes, Bogotá, Colombia. ${ }^{* *}$ Universidad del Norte, Barranquilla, Colombia, \\ y Bergische Universität Wuppertal, Alemania.
}

Doi: http://dx.doi.org/10.12804/ap134.2.2016.10

\section{Resumen}

El propósito de este estudio fue examinar el uso de la versión en español del Inventario de los Cinco Grandes (Big Five Inventory) en una muestra colombiana de conveniencia de 323 estudiantes universitarios. La estructura de los Cinco Grandes se reprodujo con un índice de congruencia factorial por encima de .90 . Al considerar la edad promedio de la muestra, el análisis se realizó aplicando el procedimiento recomendado por Soto, John, Gosling y Potter, para controlar la aquiescencia. Los análisis confirmaron diferencias por sexo. Palabras clave: Inventario de los Cinco Grandes; Colombia; diferencias de sexo en personalidad.

\section{flbstract}

The objective of the present study was to examine the use of the Big Five Inventory in its Spanish version relying on a convenience sample of 323 Colombian university students. We replicated the structure of the Big Five with a congruence factor above .90. Considering the average age of our sample, we realized our analysis applying the ipsatization procedure as suggested by Soto, John, Gosling, and Potter to control for the effect of acquiescence. Our analysis confirmed sex differences similar to those reported.

Keywords: Big Five Inventory; Colombia; sex differences in personality.

\section{Resumo}

O propósito deste estudo foi examinar o uso da versão em espanhol do Inventário dos Cinco Grandes (Big Five Inventory - BFI traduzido por Benet-Martínez \& John, 1998) em uma amostra colombiana de conveniência de 323 estudantes universitários. Replicou-se a estrutura dos Cinco Grandes com um índice de congruência fatorial por cima de .90 . Considerando a idade média da amostra, a análise se realizou aplicando o procedimento

* Elvira Salgado, Facultad de Administración, Universidad de los Andes, Bogotá, Colombia; Elvia Vargas-Trujillo, Departamento de Psicología, Universidad de los Andes; Eduardo Wills-Herrera, Facultad de Administración, Universidad de los Andes. ** Jana Schmutzler, Escuela de Negocios, Universidad del Norte, Barranquilla, Colombia, y Bergische Universität Wuppertal, Alemania. La correspondencia relacionada con este artículo debe ser dirigida a Elvira Salgado, Facultad de Administración, Universidad de los Andes, Bogotá, Colombia. Correo electrónico: esalgado@uniandes.edu.co

Cómo citar este artículo: Salgado, E., Vargas-Trujillo, E., Schmutzler, J. \& Wills-Herrera, E. (2016). Uso del Inventario de los Cinco Grandes en una muestra colombiana. Avances en Psicología Latinoamericana, 34(2), 365-382. doi: http://dx.doi. org/10.12804/apl34.2.2016.10 
recomendado por Soto, John, Gosling e Potter para controlar a aquiescência. As análises confirmaram diferenças por sexo similares.

Palavras-chave: Inventário dos Cinco Grandes; Colômbia; diferenças de sexo em personalidade.

Según John, Naumann y Soto (2008), el modelo de los Cinco Grandes se convirtió a finales de la década de los noventa en la propuesta descriptiva de la personalidad más usada en investigación. $\mathrm{Su}$ importancia proviene de su poder explicativo en el campo de la psicología, en el que se ha relacionado con variables tan diferentes como el desempeño académico (Poropat, 2009), la propensión a la accidentalidad en el trabajo o fuera de este (Clarke \& Robertson, 2005) y los comportamientos relativos a la salud (Bogg \& Roberts, 2004). Igualmente, los investigadores de las área de la psicología industrial, del trabajo y de las organizaciones lo han adoptado (por ejemplo, véase el Handbook of Personality at Work editado por Christiansen \& Tett, 2013) y han encontrado evidencia de su relación con múltiples variables de resultado, entre ellas el desempeño (Barrick \& Mount, 1991), la creatividad (Feist, 1998; Batey, Chamorro-Premuzic \& Furnham, 2010), la satisfacción (Judge, Heller \& Mount, 2002) y el surgimiento del liderazgo (Foti \& Hauenstein, 2007).

En general, se reconoce que el Inventario de los Cinco Grandes (Big Five Inventory [BFI]; John, Donahue \& Kentle, 1991) es una medida confiable y factorialmente válida del modelo que lo fundamenta. El BFI ha sido traducido al español y usado por Benet-Martínez y John (1998). A pesar de su disponibilidad en español y su relevancia para el campo de la psicología clínica, la psicología industrial y el comportamiento organizacional, las propiedades del instrumento han sido escasamente estudiadas en Latinoamérica; una necesidad clara, dadas las diferencias léxicas y semánticas con el español usado en España, por los latinos en Estados Unidos y los diferentes países latinoamericanos
(Benet-Martínez \& John, 1998). Como lo mencionan Ledesma, Sánchez y Díaz-Lázaro (2011), un adjetivo fácilmente reconocible y muy usado en un país, por ejemplo, México, puede no serlo en otro, como Argentina.

Una búsqueda en Scopus y Redalyc para los últimos años arrojó solamente tres estudios que aplican el BFI en el contexto de América Latina: Schmitt et al. (2007) usan el mismo instrumento que en el presente estudio, donde usamos una versión ligeramente modificada de la versión de Benet-Martínez y John (1998). Estos autores consideran muestras de Argentina, Bolivia, Chile, México y Perú, en un estudio de 56 países. Bleidorn et al. (2013) estudiaron una muestra de 62 países, obtenida a través de la página web del Gosling-Potter Internet Personality Project. En dicho estudio están representados 14 países latinoamericanos hispanoparlantes, entre ellos Colombia. En Argentina, Ledesma, Sánchez y Díaz-Lázaro (2011) compararon su Listado de Adjetivos para Evaluar la Personalidad (AEP) con el BFI, en una versión traducida por Castro-Solano y Casullo (2001).

Aunque estos tres estudios reprodujeron de manera exitosa la estructura de los Cinco Grandes en países de América Latina, solamente el estudio de Ledesma et al. (2011) revela inconvenientes con algunos ítems. Al mismo tiempo, se carece de información sobre el factor de congruencia para los distintos países de América Latina, dado que los estudios de Schmitt et al. (2007) y Bleidorn et al. (2013) solamente lo arrojan a nivel agregado para la región. Considerando las diferencias léxicas y semánticas mencionadas, nuestro objetivo es no solamente verificar la pertinencia de usar el BFI (John, Donahue \& Kentle, 1998) en su versión en español (Benet-Martínez \& John, 1998) para la medición de la personalidad en Colombia, sino revelar potenciales inconvenientes con ítems específicos, a efectos de proveer insumos para futuras investigaciones. Concretamente, en este estudio buscamos responder las siguientes preguntas: ¿se puede reproducir de manera satisfactoria la estructura factorial del 
BFI en su versión en español en Colombia? ¿El BFI permite verificar el perfil de los Cinco Grandes en una muestra colombiana? ¿Cuáles ítems deberían ajustarse al contexto de Colombia para mejorar la congruencia de los factores?

\section{El modelo de las cinco grandes dimensiones de la personalidad}

El término personalidad hace referencia a la manera característica en la que una persona piensa, siente, se comporta y se relaciona con otros (Widiger \& Mullins-Sweatt, 2009). Aunque el interés por las diferencias individuales ha acompañado a la humanidad desde épocas primitivas (Millon, 2012) solo en los últimos treinta años el campo de la psicología de la personalidad se consolidó como área independiente ${ }^{1}$ (Barenbaum \& Winter, 2008; John, Robins \& Pervin, 2008).

John y Srivastava (2001) señalan que este campo de la disciplina se caracteriza por la diversidad de aproximaciones a la comprensión, explicación y estudio de las diferencias individuales. A modo de síntesis, en la literatura es posible diferenciar las perspectivas que distinguen rasgos o patrones consistentes de comportamiento (v.g., Allport, 1937; Cattell, 1943), las que identifican disposiciones o motivaciones (v.g., Murray, 1938; Ryan \& Decy, 2000), las que se centran en las cogniciones (v.g., Bandura, 2001; Mischel \& Shoda, 1995), las que examinan la influencia del contexto social y cultural (v.g., Nisbett, 2003; Markus \& Kitayama, 1991) y las que consideran mecanismos biológicos o evolutivos (v.g., DeYoung \& Gray, 2009; Zuckerman, 1991).

Hoy en día, uno de los marcos de referencia más influyentes en el estudio de la personalidad es el de los Cinco Grandes. Este modelo organiza en cinco dimensiones los aspectos del individuo que son

1 En la American Psychological Association, la personalidad es un tema de las divisiones 8 (sociedad para la personalidad y la psicología social) y 20 (desarrollo adulto y envejecimiento). estables a lo largo del ciclo vital y que determinan la manera como el individuo tiende a responder a las demandas de la vida cotidiana (Gerber, Huber, Doherty \& Dowling, 2011). El origen de este modelo se remonta a los primeros trabajos sobre el tema en el campo de la psicología que partían de la proposición de que las características más visibles y socialmente relevantes de la personalidad se encuentran codificadas en las lenguas naturales (véase la historia del modelo en John \& Srivastava, 2001; John et al., 2008a).

Con base en este planteamiento, a partir de un cuidadoso análisis léxico, se logró establecer un modelo descriptivo de la personalidad, es decir, una taxonomía. Para tal fin, equipos de investigación independientes obtuvieron listas de adjetivos o frases que pueden usarse para describir características individuales perdurables, es decir, los rasgos de personalidad. Esos listados se los presentaban a los participantes en sus estudios, quienes debían valorar qué tan bien cada palabra o frase les permitía describir a otras personas. Posteriormente, mediante análisis factoriales, los investigadores identificaban el número de dimensiones necesarias para explicar los datos. De esta manera, se obtuvo evidencia de que las respuestas podían agruparse en cinco dimensiones (Tupes \& Christal, 1961): extraversión, afabilidad, meticulosidad, estabilidad emocional (denominado por otros neuroticismo) y apertura a la experiencia.

Siguiendo las palabras de John et al. (2008a), a continuación se definen las dimensiones de los Cinco Grandes: (1) apertura a la experiencia (openness to experience), que "describe la amplitud, profundidad, originalidad y complejidad de la vida mental y aquella derivada de la experiencia de una persona"; (2) meticulosidad (conscientiousness), que "describe el control de los impulsos que es prescrito socialmente y facilita los comportamientos dirigidos al logro de tareas y metas, como pensar antes de actuar, postergar las recompensas, seguir normas y reglas, y planear, organizar y priorizar las tareas"; (3) extroversión (extraversion), 
que "implica una aproximación energética hacia el mundo social y material e incluye rasgos como sociabilidad, actividad, asertividad y emocionalidad positiva"; (4) afabilidad (agreeableness), que "contrasta una orientación prosocial y comunal hacia los otros con el antagonismo, e incluye rasgos como altruismo, ternura, confianza y modestia", y (5) neuroticismo (neuroticism), ${ }^{2}$ que "contrasta la estabilidad emocional y un ánimo estable con la emocionalidad negativa, tal como sentirse ansioso, nervioso, triste y tenso" (p. 120).

Esta estructura de cinco factores se ha reproducido, con diversas muestras, en una gran variedad de lenguajes y contextos culturales (John et al., 2008a). Fue Goldberg (1981) quien denominó la taxonomía derivada de sus análisis los Cinco Grandes. Según John et al. (2008a) su intención era hacer hincapié en que estas dimensiones representan la personalidad en un nivel alto de abstracción y por ello cada una puede resumir distintos y numerosos rasgos.

John et al. (2008a) afirman que la estructura de los Cinco Grandes no representa una perspectiva teórica particular, sino una taxonomía que cumple una función integradora, al representar bajo un marco común diferentes "conjuntos específicos de características de personalidad relacionadas entre sí, en vez de examinar de manera separada los miles de atributos particulares que hacen a los seres humanos individuales y únicos" (p. 116).

\section{El Inventario de los Cinco Grandes}

Para medir la estructura de cinco grandes dimensiones de la personalidad existen diferentes

2 Diversos autores han llamado esta dimensión de diferentes maneras y los autores del BIF (John, Donahue \& Kentle, 1991; John et al., 2008a) prefieren el término neuroticism. La palabra neuroticismo no es la más adecuada en español, ya que no solo no existe, sino que por su etimología no expresa convenientemente el significado que se quiere expresar en inglés. Sin embargo, la usamos dado que ya se usa esta palabra en la literatura surgida en nuestros países. instrumentos. Los tres más usados son: Trait Descriptive Adjectives (Goldberg, 1992), Neo Personality Inventory (Costa \& McCrae, 1985) y el BFI (John et al., 1991; John \& Srivastava, 2001). Cada uno ha demostrado tener niveles altos de confiabilidad y validez predictiva. Si bien la convergencia entre estos instrumentos es alta, no es perfecta, ya que no todos tienen el mismo contenido. El uso de uno u otro, según recomendación de John et al. (2008a), depende de los objetivos buscados con su aplicación, las personas que lo van a responder y el tiempo disponible.

El BFI está compuesto por 44 ítems que consisten de frases cortas y fáciles de entender. Así, se retiene la ventaja de los adjetivos como brevedad y simplicidad, evitando sus desventajas como ambigüedad o definiciones múltiples y deseabilidad aparente (Benet-Martínez \& John, 1998). En algunos casos, se añade información sobre el contexto o se aclaran los adjetivos. Los ítems van antecedidos de la afirmación: "Me veo a mí mismo como alguien que..." y termina con una frase corta como: “... es original, tiene nuevas ideas". Los encuestados responden con base en una escala Likert de 5 puntos que va desde "totalmente en desacuerdo" hasta "totalmente de acuerdo". Estas escalas han mostrado alta consistencia interna, confiabilidad en el retesteo, una clara estructura factorial, así como una convergencia fuerte con versiones más extensas usadas para medir los Cinco Grandes, lo que favorece su uso (Soto, John, Gosling \& Potter, 2008).

\section{La investigación transcultural}

Desde la perspectiva de imposed-etic ${ }^{3}$ (Berry, 1980), los Cinco Grandes se han investigado en

3 En una perspectiva emic, se partiría de la elaboración de la lista en el lenguaje del investigador de los adjetivos representativos de rasgos que naturalmente surgen y que no se someten a un esfuerzo de representar estructuras en otras lenguas a partir de taxonomías o modelos existentes. La lista definitiva podría, entonces, no converger exactamente 
muchas lenguas y países del mundo, y con diferentes instrumentos de medición (por ejemplo, Bleidorn et al., 2013; Costa, Terraciano \& McCrae, 2001; Ledesma et al., 2011; McCrae et al., 2005; Schmitt et al., 2007; Schmitt, Realo, Voracek \& Allik, 2008; Ortiz et al., 2007). Eso quiere decir que el instrumento existente traducido a las diferentes lenguas se aplica en diversos países para analizar la generalización transcultural, más que su generalización translingüística. Dado que las estructuras encontradas son muy similares, se puede decir que el instrumento es poco específico culturalmente.

Inicialmente, el BFI fue traducido al español por Benet-Martínez y John (1998). En este estudio se usó la versión en español del proyecto ISDP (Schmitt et al., 2007). Entre las dos versiones existen unas pocas diferencias que mencionaremos más adelante.

\section{La influencia de la edad}

Dos variables sociodemográficas muy estudiadas que pueden influir en la estructura de los Cinco Grandes son la edad y el sexo. Varias investigaciones sugieren que, en general, la personalidad cambia hacia una mayor madurez, pero este proceso puede diferir dependiendo del contexto cultural. Por lo tanto, es importante tomar en cuenta la edad en un estudio como el nuestro.

Se pueden distinguir varias explicaciones a este fenómeno. McCrae y Costa (1992) reportan que, a partir de la adolescencia tardía, aumenta la afabilidad y la meticulosidad y decrecen las otras tres dimensiones, un patrón similar a través de diferentes culturas. Los autores proponen que estos cambios con la edad tienen una base biológica, aunque también pueden estar ocultando divergencias en las dimensiones evaluadas. La hipótesis de que la tasa de cambio decrece con la edad no fue sustentada

con las dimensiones propuestas en inglés. Un esfuerzo con métodos mixtos en América Latina es el de Ledesma et al. (2011) a partir del FFM y el de Ortiz et al. (2007). de manera consistente con los datos de McCrae et al. (2004). En un reciente artículo, Bleidorn et al. (2013) confirmaron incrementos significativos en tres de las dimensiones - afabilidad, meticulosidad y apertura a la experiencia - y disminuciones significativas en neuroticismo y en extroversión. Estos resultados sugieren que la maduración es un fenómeno universal, aun cuando se presentan diferencias en la edad a la que ocurre.

Para Bleidorn et al. (2013), estas diferencias se deben a una maduración de orden social. Así que estos resultados pueden interpretarse a la luz de dos teorías: la de la inversión social (Lodi-Smith \& Roberts, 2007) y la de la adultez emergente (Arnett, 2000). En este sentido, Roberts, Wood y Smith (2005) plantean que las personas invertimos en y nos comprometemos con roles sociales adultos que tienen que ver con el trabajo, la familia, la religión y el trabajo voluntario. Por ejemplo, en lo que respecta al trabajo, hacemos una inversión social al autodefinirnos con un rol particular y comprometernos con una carrera profesional. En sus investigaciones han encontrado que los esfuerzos en inversión social están positivamente relacionados con aumentos en afabilidad, meticulosidad y estabilidad emocional.

Arnett (2000), por su parte, propone un periodo diferente a la adolescencia o a la adultez, que llama adultez emergente, para los jóvenes de 18 a 25 años de sociedades desarrolladas en Occidente. En este estadio, los jóvenes no tienen que ajustarse a roles sociales y a expectativas normativas. Ellos viven más bien un periodo de exploración de su identidad de manera autónoma sin necesidad de comprometerse. Estos jóvenes, en la mayoría de los casos, continúan sus estudios y aunque dependan de sus padres económicamente, no viven en la casa paterna. Según Arnett (2000), esta cohorte es la más inestable en cuanto a su sitio de residencia, subjetivamente no se sienten adultos y buscan su identidad en los tres aspectos más importantes del paso a la vida adulta: el amor, el trabajo y la visión del mundo. 
El estudio de Bleidorn et al. (2013) encuentra sustento para los planteamientos de la teoría de la inversión social al identificar cambios tempranos en las tres dimensiones que ellos habían propuesto en estudios previos (aumentos en afabilidad, meticulosidad y decrecimiento en neuroticismo); pero solo en aquellas culturas donde se da un paso temprano normativo al trabajo. Al mismo tiempo, el efecto de la transición en roles de familia no tuvo el efecto esperado.

Desde una perspectiva contextual o ecológica, los cambios de maduración también pueden ser el resultado de la interacción compleja que se establece entre las características biológicas del individuo y múltiples factores del medio social en el que nace y vive (Srivastava, John, Gosling \& Potter, 2003). Estos investigadores encontraron que los patrones de cambio son complejos, ya que no solo las diversas dimensiones varían a tasas diferentes en momentos distintos, sino que interactúan con el sexo. Por otra parte, Soto, John, Gosling y Potter (2010), con una muestra de individuos entre los 10 y los 65 años de edad, obtuvieron evidencia de ese panorama complejo, si bien no logran confirmar que las diferencias observadas se deban a la interacción de factores biológicos y sociales. Al tener en cuenta la infancia y adolescencia que no se había considerado en trabajos previos, encuentran tendencias curvilíneas con patrones diferentes en las distintas dimensiones y que el efecto del sexo se empieza a vislumbrar desde la adolescencia.

Por último, existe una explicación de carácter metodológico, según la cual las relaciones entre edad y dimensiones de personalidad pueden deberse parcialmente al efecto de sesgos de respuesta, como la aquiescencia (Soto, John, Gosling \& Potter, 2008) y la deseabilidad social (Soubelet \& Salthouse, 2011). Vigil-Colet, Morales-Vives y Lorenzo-Seva (2013) encuentran que, efectivamente, ambos sesgos aumentan con la edad y al corregirlos se observa un decrecimiento mayor al observado en meticulosidad y afabilidad en gente mayor, en interacción con el sexo. Por otro lado, la confusión entre el contenido de personalidad propio del cuestionario y el estilo de respuesta puede disminuir la validez. En un artículo más reciente, Soto et al. (2010) controlan la aquiescencia en una muestra con edades de 10 a 65 años, debido a que esta puede generar un aumento significativo en la correlación entre ítems. Este aumento puede alterar la confiabilidad de los datos, ya que la consistencia interna se verá artificialmente inflada.

\section{La influencia del sexo}

Adicional a la edad, se han encontrado diferencias en las cinco dimensiones de la personalidad entre hombres y mujeres. En un principio, los autores evaluaron rasgos diversos y empezaron a encontrar diferencias por sexo (Feingold, 1994). Costa y McCrae (1992) hallaron mayores promedios para mujeres en las dimensiones de neuroticismo y afabilidad (usando el NEO Personality Inventory-Revised [NEO-PI-R]). Feingold (1994) encontró en un metanálisis que los hombres son más asertivos y con una autoestima ligeramente más alta que las mujeres, siendo estas más extrovertidas, ansiosas, confiadas y, en especial, tiernas. Benet-Martínez y John (1998) encontraron estas mismas diferencias "pequeñas pero consistentes" (p. 732) en neuroticismo y afabilidad usando el BFI y el NEO Five Factor Inventory [NEO-FFI] en español e inglés. Igualmente, Costa et al. (2001), en un estudio que abarcó 26 naciones (solo un país latinoamericano, Perú), usando el NEO-PI-R encontraron diferencias significativas entre hombres y mujeres en algunas de las facetas de la dimensión extroversión. Un resultado similar se observó en apertura a la experiencia.

Schmitt et al. (2008) estudiaron las diferencias por sexo usando el BFI en 55 países diferentes y encontraron que, en general, los puntajes son superiores para las mujeres en las dimensiones de neuroticismo, afabilidad, meticulosidad y extroversión, y menores en apertura a la experiencia. Bleidorn et al. (2013) encontraron las mismas diferencias en 
su muestra de 62 países. Cabe aclarar que estas no son idénticas en todas las culturas y según Schmitt et al. (2008), a mayor riqueza nacional e igualdad entre los sexos, las diferencias tienden a ser más altas.

Entre las razones expuestas para estas diferencias se destacan tres. Desde la perspectiva biológica o evolucionista, las diferencias en personalidad entre los sexos se dan en aquellos campos donde hombres y mujeres han enfrentado problemas de adaptación, es decir, se deben a presiones de selección (Buss \& Kenrick, 1998). La segunda explicación se fundamenta en la teoría del rol social, que plantea que las diferencias entre los sexos se dan por los diferentes procesos de socialización del género, es decir, las normas y expectativas sociales que establecen los distintos roles para mujeres y hombres (Eagly, 1987). Por último, se plantea que la diferencia no es real, sino producto de artificios metodológicos: errores en la medición, sesgos como la aquiescencia o la deseabilidad social, uso de diferentes marcos de referencia para la autodescripción en diferentes culturas y procesos de atribución (Schmitt et al., 2008).

No obstante, Schmitt et al. (2008) argumentan que este tipo de explicaciones consideradas separadamente no podría explicar la brecha que se observa entre los sexos en sociedades en las que por el creciente desarrollo humano y social las oportunidades de las mujeres se acercan cada vez más a las de los hombres. Ellos proponen que las diferencias se deben a la interacción genes-entorno, es decir, entre el componente genético de la personalidad y las presiones del entorno y los eventos de la vida individual, ya que los aspectos metodológicos se pueden controlar y aun después de hacerlo las diferencias persisten.

\section{Método}

\section{Participantes}

Se conformó una muestra de conveniencia de 323 estudiantes de pregrado en una universidad privada de Bogotá (Colombia). Los datos se recabaron en el contexto de la fase 2 del International Sexuality Description Project-2 (ISDP-2) (Schmitt et al., 2002, 2007). El 55\% de los participantes eran hombres y la edad de la muestra oscila entre los 17 y los 25 años $(M=20.14 ; D T=1.51)$. En el momento de diligenciar los instrumentos, los estudiantes estaban registrados en nueve facultades, la mayoría pertenecía a Administración (39\%) e Ingeniería (34\%). El 45\% de los participantes reportó pertenecer a nivel socioeconómico medio alto, y el $41 \%$, a nivel socioeconómico alto.

\section{Instrumento}

Se empleó la versión en español del BFI (John et al., 1991) proveniente del proyecto ISDP, que es una versión ligeramente diferente a la traducida al español por Benet-Martínez y John (1998), a las diferentes lenguas de los países participantes por el método de traducir y traducir de vuelta (translation and back-translation). Los líderes del proyecto lo escogieron por su brevedad, facilidad de administración y su probada utilidad en la investigación transcultural y translingüística (Schmitt et al., 2007). Aunque en muchos ítems hay cambios menores, solamente en dos casos estos hacen que los ítems sean en su sentido diferentes a la encuesta de Benet-Martínez y John (1998). Los ítems y los efectos de estas diferencias se analizarán en detalle en los resultados (véase también tabla 3 ).

Adicionalmente, el cuestionario usado incluía una sección para medir las variables sociodemográficas: sexo, edad, programa en el cual estaban registrados los estudiantes, nivel socioeconómico y otras variables no reportadas aquí.

\section{Procedimiento}

A los estudiantes se les contactó en cursos de dos programas diferentes (Administración y Psicología), abiertos a estudiantes de toda la universidad, es decir, no eran cursos específicos de estas dos 
carreras. Se invitó a los estudiantes a participar en el estudio y los que accedieron a participar voluntariamente, después de firmar un consentimiento informado, diligenciaron el cuestionario de autorreporte durante un periodo de clase, sin recibir ningún tipo de compensación por responderlo. Un asistente graduado entrenado estuvo presente y explicó los objetivos y el procedimiento del estudio, así como el carácter voluntario, confidencial y anónimo de la participación. Adicionalmente, explicó que no había respuestas correctas o incorrectas, y estuvo atento a responder inquietudes si las hubiera. Con anterioridad a la aplicación del cuestionario, el equipo de investigación obtuvo el permiso del Comité de Ética de la Universidad para proceder con el estudio. La tasa de participación fue de1 99\%. Se excluyeron tres cuestionarios de los análisis, por el alto número de datos faltantes.

\section{Análisis de los datos}

Los análisis estadísticos descriptivos y multivariados se realizaron usando el paquete estadístico SPSS 19.0 para Windows. Teniendo en cuenta la edad promedio de la muestra de estudiantes y los resultados de Soto et al. (2008), decidimos controlar el efecto de la edad en la correlación entre ítems (de potencialmente aumentarla) mediante el procedimiento de ipsatization. Este permite estandarizar los datos de cada persona (within-person). La transformación se hace de manera que el conjunto de los datos de cada participante posea el mismo promedio y desviación estándar. Se logra al substraer primero la respuesta promedio de cada participante (obteniendo un promedio de todas sus respuestas) de cada una de sus respuestas y luego dividiendo estas diferencias por la desviación estándar de ese mismo participante. Soto et al. (2008) sugieren calcular el promedio y la desviación estándar de cada persona usando 16 pares de ítems opuestos, ya que el cuestionario no tiene el mismo número de ítems redactados para ser verdaderos o falsos (totalmente de acuerdo o en desacuerdo).
Esto puede generar una confusión entre el contenido de la personalidad propio del cuestionario y el sentido del ítem (item keying). Así, se computa un puntaje de aquiescencia - a fin de obtener el promedio de los 16 pares, es decir, 32 ítems - y luego se calcula un puntaje de extremismo en las respuestas (response extremeness) para lograr la desviación estándar de los 16 pares, es decir, 32 ítems (en la tabla 1 se señalan los pares). Los valores así derivados se aplicaron a los 44 ítems de cada individuo.

Usando estos valores estandarizados (ipsatized values), se corrió un análisis de componentes principales (ACP) con rotación varimax, que ha sido el método más usado para estudiar la estructura de la personalidad (Soto et al., 2008), ya que desde 1994 Church y Burke identificaron que el análisis factorial confirmatorio de correspondencia no parece ser el método óptimo. Aunque la muestra original fue de 323 individuos, el ACP se hizo con 305, porque se usó el procedimiento de eliminar los casos con valores faltantes. Esta muestra es adecuada, ya que se tiene un poco más del mínimo estipulado por Hair, Anderson, Tatham y Black (1995), de 5 casos por ítem, y con esta podemos considerar significativas cargas por encima de .35. La medida de adecuación muestral de Kaiser-Meyer-Olkin superó el mínimo valor aceptable de .60 (.79) (Kaiser, 1970) y la prueba de esfericidad de Bartlett (1951) fue significativa $(p=0,00)$, proporcionando así el soporte para la factorabilidad de la matriz de correlaciones. Adicionalmente, para examinar la hipótesis de diferencias por sexo se llevó a cabo un análisis de varianza (Anova).

\section{Resultados}

\section{Equivalencia estructural de los Cinco Grandes}

Nuestros resultados muestran que se reproduce una solución de cinco factores, indicada por un corte claro en el Scree Plot en el quinto componente. Además, la mayoría de ítems cargaron en los 
factores previamente propuestos, lo cual explica el $42 \%$ de la varianza. Se obtuvo una estructura bastante clara: con la excepción de cinco ítems, que se analizarán en detalle más adelante, todos los otros cargaron en el factor adecuado - con cargas superiores a .35 - sin presentar una carga importante en otro factor. ${ }^{4}$ En la tabla 1 se pre- senta la estructura factorial resultante, así como el ítem correspondiente en la encuesta usada por Benet-Martínez y John (1998).

En el análisis de congruencia factorial usando la fórmula de Tucker (1951) — con las cargas de todos los ítems que se supone conforman cada factor, independientemente de que hubieran o no

Tabla 1

Estructura factorial de los Cinco Grandes de Personalidad

\begin{tabular}{|c|c|c|c|c|c|c|c|}
\hline Ítems encuesta & $\begin{array}{l}\text { Apertura a la } \\
\text { experiencia }\end{array}$ & $\begin{array}{l}\text { Meticu- } \\
\text { losidad }\end{array}$ & $\begin{array}{l}\text { Extro- } \\
\text { versión }\end{array}$ & $\begin{array}{l}\text { Afabi- } \\
\text { lidad }\end{array}$ & $\begin{array}{l}\text { Neuroti- } \\
\text { cismo }\end{array}$ & Pareja* & $\begin{array}{l}\text { Item B-M } \\
\qquad \& \mathrm{~J}^{* *}\end{array}$ \\
\hline Es relajado/a, maneja bien el estrés & & & & & -.70 & 12 & 9 \\
\hline $\begin{array}{l}\text { Es emocionalmente estable (no se altera con } \\
\text { facilidad) }\end{array}$ & & & & & -.66 & 13 & 19 \\
\hline Permanece tranquilo/a en las situaciones tensas & & & & & -.64 & 14 & 35 \\
\hline Es depresivo/a triste & & & & & .60 & & 4 \\
\hline Se preocupa bastante & & & & & .58 & 12 & 26 \\
\hline Puede sentirse tenso/a & & & & & .57 & & 15 \\
\hline Se pone nervioso/a fácilmente & & & & & .47 & 14 & 38 \\
\hline Puede tener mal genio & & & & -.42 & .41 & 13 & 30 \\
\hline Tiende a ser desorganizado/a & & -.65 & & & & 10 & 18 \\
\hline Tiende a ser perezoso/a & & -.64 & & & & 11 & 25 \\
\hline Hace las cosas de manera eficiente & & .62 & & & & 10 & 29 \\
\hline Es cuidadoso/a en su trabajo & & .60 & & & & 8 & 3 \\
\hline Se distrae fácilmente & & -.59 & & & & 8 & 42 \\
\hline Persevera hasta que la tarea se haya terminado & & .59 & & & & 11 & 21 \\
\hline Puede ser algo descuidado/a & & -.58 & & & & 9 & 8 \\
\hline Hace planes y los saca adelante & & .51 & & & & & 34 \\
\hline Es un/a trabajador/a de fiar & & .42 & & & & 9 & 14 \\
\hline Es extrovertido/a sociable & & & .79 & & & 3 & 43 \\
\hline Es algo tímido/a, inhibido/a & & & -.74 & & & 3 & 27 \\
\hline Es hablador/a & & & .73 & & & 1 & 1 \\
\hline Es reservado/a & & & -.64 & & & 2 & 6 \\
\hline
\end{tabular}

4 Hair et al. (1995) plantean que con una muestra superior a 250 individuos, una carga de .35 puede ser considerada significativa, con un nivel de poder de $80 \%$ y un nivel de significancia de .05 . 


\begin{tabular}{|c|c|c|c|c|c|c|c|}
\hline Ítems encuesta & $\begin{array}{l}\text { Apertura a la } \\
\text { experiencia }\end{array}$ & $\begin{array}{l}\text { Meticu- } \\
\text { losidad }\end{array}$ & $\begin{array}{l}\text { Extro- } \\
\text { versión }\end{array}$ & $\begin{array}{l}\text { Afabi- } \\
\text { lidad }\end{array}$ & $\begin{array}{l}\text { Neuroti- } \\
\text { cismo }\end{array}$ & Pareja* & $\begin{array}{l}\text { Item B-M } \\
\qquad \& \mathrm{~J}^{* *}\end{array}$ \\
\hline Genera mucho entusiasmo & & & .59 & & & 2 & 32 \\
\hline Está lleno/a de energía & & & .53 & & & & 11 \\
\hline Tiende a ser tranquilo/a & & & -.18 & & -.67 & 1 & 16 \\
\hline $\begin{array}{l}\text { Defiende sus derechos y expresa su opinión sin } \\
\text { agredir a los demás }\end{array}$ & & & .22 & .33 & & & 40 \\
\hline Es refinado/a en arte, música, literatura & .63 & & & & & & 39 \\
\hline Tiene inventiva & .62 & & & & & & 23 \\
\hline Es ingenioso/a, un/a pensador/a profundo/a & .61 & & & & & & 31 \\
\hline Tiene pocos intereses artísticos & -.60 & & & & & 16 & 44 \\
\hline Es original, tiene ideas nuevas & .57 & & & & & 15 & 5 \\
\hline Valora las experiencias artísticas y estéticas & .55 & & & & & 16 & 17 \\
\hline Tiene una imaginación activa & .54 & & & & & & 20 \\
\hline Le gusta reflexionar, jugar con las ideas & .52 & & & & & & 36 \\
\hline Tiene curiosidad por cosas muy diferentes & .51 & & & & & & 10 \\
\hline Prefiere trabajo rutinario & -.46 & & & & & 15 & 12 \\
\hline Es considerado/a y amable con casi todo el mundo & & & & .60 & & 7 & 37 \\
\hline Es a veces descortés con los demás & & & & -.55 & & 7 & 22 \\
\hline Actúa desinteresadamente con los demás y les ayuda & & & & .50 & & 5 & 7 \\
\hline Tiende a encontrar defectos en los demás & & & & -.50 & & 4 & 2 \\
\hline Perdona fácilmente & & & & .49 & & 4 & 28 \\
\hline Le gusta cooperar con los demás & & & & .45 & & 6 & 41 \\
\hline Suele reñir con los demás & & & & -.39 & & 5 & 13 \\
\hline Puede ser frío/a y distante & & & -.42 & -.46 & & 6 & 33 \\
\hline Es generalmente confiado/a & & -.20 & .10 & .25 & -.29 & & 24 \\
\hline Coeficiente de Tucker de congruencia factorial & .95 & .99 & .93 & .98 & .98 & & \\
\hline
\end{tabular}

Nota. Esta estructura se logró con un ACP y rotación varimax. Solo se muestran las cargas principales, salvo que un ítem haya cargado en dos o más factores con cargas similares. Los ítems del cuestionario escritos en fuente normal pertenecen al factor y cargan adecuadamente. A continuación en un factor se presenta en negrilla el ítem que no cargó adecuadamente. En cursivas van los ítems a los que se les hizo algún cambio en la redacción con relación a la encuesta enviada por el investigador principal del ISDP -2 (véase tabla 3).

* En esta fila va la numeración que indica cuáles son parejas para el procedimiento de estandarización.

** En esta fila va el número correspondiente al ítem en el cuestionario de Benet-Martínez y John (1998).

logrado una carga suficientemente alta - cuatro factores tienen índices por encima de .95 , lo que según Lorenzo-Seva y Ten Berge (2006) significa que son estadísticamente iguales. Extroversión, con un factor de .93 , tiene una similitud razonable según estos autores. A continuación se presenta la conformación de cada factor y los problemas que se presentaron con esta muestra:

Para dos de los cinco factores - apertura a la experiencia y meticulosidad - los diferentes ítems cargaron según lo esperado, con cargas superiores a .40. Para los otros tres factores, identificamos 
unos ítems con problemas, ya fuere por cargas en dos dimensiones o cargas en la dimensión no esperada. Como se observa en la tabla 1, en el caso de extroversión, seis ítems cargaron debidamente y con dos ("Tiende a ser tranquilo" y "Defiende sus derechos y expresa su opinión sin agredir a los demás") se presentaron problemas, de manera que se excluyeron. De los nueve ítems que debían cargar en afabilidad, siete lo hicieron debidamente; los otros dos ("Puede ser frío y distante" y "Es generalmente confiado") se excluyeron. De los ocho ítems que debían cargar en el factor neuroticismo, siete lo hicieron debidamente; pero el ítem "Puede tener mal genio" se excluyó. Además, un ítem inicialmente perteneciente a la dimensión de extroversión ("Tiende a ser tranquilo") cargó muy bien en esta dimensión, de manera que se añade. Así, este factor cuenta con ocho ítems.

En la tabla 2 se presentan los descriptivos de los cinco factores. Los coeficientes alfa de 4 están por encima de .70, valor mínimo sugerido como buen indicador de esta medida (Ghiselli, Campbell \& Zedeck, 1981). La excepción es el caso de afabilidad, lo cual no es extraño, dadas las dificultades que tuvimos con varios de sus ítems. Es de anotar que tanto Ledesma et al. (2011) en su estudio usando el BFI en Argentina como Schmitt et al. (2007), en su estudio internacional, enfrentaron el mismo problema.

Por último, respecto a las diferencias por sexo, encontramos puntajes superiores para las mujeres en las dimensiones de neuroticismo, afabilidad, meticulosidad y extroversión, y menores en apertura a la experiencia, que son las mismas reportadas por Schmitt et al. (2008), como promedio de las diferencias entre las diferentes culturas. Todas estas diferencias fueron muy pequeñas, aun cuando la de apertura es la única estadísticamente significativa $(p=.04)$.

\section{Discusión}

En este artículo se presentan los resultados del uso del BFI en una muestra de estudiantes universitarios en Colombia. Si bien en la base de datos de Bleidorn et al. (2013) se incluyen datos colombianos, en la literatura disponible no se encontró evidencia que dé cuenta del uso del modelo de las Cinco Grandes dimensiones de la personalidad en el país. Contar con información a este respecto es importante porque, como se mencionó, el modelo de los Cinco Grandes es el más usado en la actualidad en muchos de los campos de la psicología, sobre todo en el área de la psicología del trabajo y las organizaciones.

Con los datos examinados, se logró reproducir la estructura factorial esperada para el BFI, con cargas altas en cada dimensión y un índice de congruencia muy alto. No obstante, se identificaron dificultades con algunos ítems específicos en los factores de extroversión, afabilidad y neuroticismo, que dan lugar a una mejora de este instrumento para el caso colombiano. Los alfas de Cronbach de cuatro de las dimensiones también son aceptables,

Tabla 2

Estadísticas descriptivas de los Cinco Grandes de Personalidad

\begin{tabular}{|c|c|c|c|c|c|c|c|c|c|c|}
\hline & Ítems & Mín. & Máx. & Promedio & Des. Est. & 1 & 2 & 3 & 4 & 5 \\
\hline Apertura & 10 & 1.70 & 5.00 & 3.72 & .63 & .81 & & & & \\
\hline Meticulosidad & 9 & 1.89 & 4.89 & 3.38 & .58 & $.14^{*}$ & .76 & & & \\
\hline Extroversión & 6 & 1.17 & 5.00 & 3.36 & .76 & $.24 * *$ & $.16^{* *}$ & .84 & & \\
\hline Afabilidad & 7 & 1.50 & 4.83 & 3.54 & .55 & $.16^{* *}$ & $.19 * *$ & $.12 *$ & .59 & \\
\hline Neuroticismo & 8 & 1.25 & 4.63 & 3.11 & .72 & .08 & $.14^{*}$ & $.25^{* *}$ & .10 & .80 \\
\hline
\end{tabular}

$N=320$. Las correlaciones con $*$ son significativas al $.05 \mathrm{y}$ con $* *$ son significativas al .01. En la diagonal se encuentra el alfa de Cronbach. 
y tal como lo plantean otros autores, se obtuvo un alfa bajo en afabilidad.

Presentamos en la tabla 3 los ítems que tuvieron problemas. En el aparecen la versión usada en este trabajo, la versión de Benet-Martínez y John (1998) y la versión original del inglés (John et al., 1991). Con base en los resultados del análisis, en la siguiente columna incluimos la versión que sugerimos usar en futuras oportunidades. Adicionalmente, incluimos en la última columna la definición según la Real Academia Española (RAE, 2001) de los diferentes ítems.

Para tres de los cinco ítems problemáticos ("Tiende a ser tranquilo", "Puede ser frío y distante" y "Puede tener mal genio") podemos identificar un problema de traducción de la encuesta usada en comparación con la versión de Benet-Martínez y John (1998). En los tres casos, nuestro argumento está sustentado, ya que los ítems no fueron identi- ficados por los estudiantes como el par opuesto de los ítems propuestos por Soto et al. (2008). "Tiende a ser tranquilo", usado en la presente investigación, tiene una connotación diferente a "callado", como se usa en la versión de Benet-Martínez y John (1998), y por ello no fue calificado como el opuesto de "es hablador/a". Cargó muy bien en neuroticismo, ya que podría ser el contrario de "se pone nervioso fácilmente". En el caso del ítem "Puede ser frío y distante", sus dos términos pueden referirse a una persona poco sociable, sin mucha energía, inhibida, es decir, rasgos primarios de la extroversión. Nuevamente, los estudiantes no lo percibieron como opuesto al par "Le gusta cooperar con los demás", lo que sustenta nuestro argumento. Por último, el ítem original de Benet-Martínez y John (1998): "Es temperamental, de humor cambiante" no es equivalente a "Tener mal genio", es decir, mal carácter o temperamento

Tabla 3

Versiones diferentes de items problemáticos

\begin{tabular}{|c|c|c|c|c|}
\hline Ítems-encuesta & $\begin{array}{c}\text { Benet-Martínez y } \\
\text { John (1998) }\end{array}$ & John et al. (1991) & Ítem recomendado & RAE (2001) \\
\hline $\begin{array}{l}\text { Tiende a ser tran- } \\
\text { quilo/a }\end{array}$ & $\begin{array}{l}\text { Tiende a ser ca- } \\
\text { llado }\end{array}$ & Tends to be quiet & $\begin{array}{l}\text { Tiende a ser ca- } \\
\text { llado }\end{array}$ & $\begin{array}{l}\text { Quieto, sosegado, pacífico. Dicho de una } \\
\text { persona que se toma las cosas con tiempo, } \\
\text { sin nerviosismos ni agobios, y que no se } \\
\text { preocupa por quedar bien o mal ante la } \\
\text { opinión de los demás. }\end{array}$ \\
\hline $\begin{array}{l}\text { Defiende sus dere- } \\
\text { chos y expresa su } \\
\text { opinión sin agredir } \\
\text { a los demás }\end{array}$ & $\begin{array}{l}\text { Es asertivo, no } \\
\text { teme expresar lo } \\
\text { que quiere }\end{array}$ & $\begin{array}{l}\text { Has an assertive } \\
\text { personality }\end{array}$ & $\begin{array}{l}\text { Defiende sus de- } \\
\text { rechos y no teme } \\
\text { expresar lo que } \\
\text { quiere }\end{array}$ & Afirmativo \\
\hline $\begin{array}{l}\text { Puede ser frío/a y } \\
\text { distante }\end{array}$ & $\begin{array}{l}\text { Es a veces frío y } \\
\text { distante }\end{array}$ & $\begin{array}{l}\text { Can be cold and } \\
\text { aloof }\end{array}$ & $\begin{array}{l}\text { Es a veces frío y } \\
\text { distante }\end{array}$ & $\begin{array}{l}\text { Frío, a: Que, respecto de una persona o } \\
\text { cosa, muestra indiferencia, desapego o } \\
\text { desafecto, o que no toma interés por ella. }\end{array}$ \\
\hline & & & & $\begin{array}{l}\text { Distante: dicho de una persona que rehúye } \\
\text { el trato amistoso o la intimidad. }\end{array}$ \\
\hline $\begin{array}{l}\text { Es generalmente } \\
\text { confiado/a }\end{array}$ & $\begin{array}{l}\text { Es generalmente } \\
\text { confiado }\end{array}$ & $\begin{array}{l}\text { Is generally trus- } \\
\text { ting }\end{array}$ & $\begin{array}{l}\text { Es una persona } \\
\text { que en general } \\
\text { confía en los otros }\end{array}$ & $\begin{array}{l}\text { Crédulo, imprevisor. Presumido, satisfe- } \\
\text { cho de sí mismo. }\end{array}$ \\
\hline $\begin{array}{l}\text { Puede tener mal } \\
\text { genio }\end{array}$ & $\begin{array}{l}\text { Es temperamental, } \\
\text { de humor cam- } \\
\text { biante }\end{array}$ & Can be moody & $\begin{array}{l}\text { Es temperamental, } \\
\text { de humor cam- } \\
\text { biante }\end{array}$ & Mal carácter, temperamento difícil. \\
\hline
\end{tabular}


difícil, como lo define la RAE (2001). Por ello tampoco funcionó bien como par opuesto de "Es emocionalmente estable".

Creemos que para los otros dos ítems problemáticos ("Defiende sus derechos y expresa su opinión sin agredir a los demás" y "Es generalmente confiado"), el contexto colombiano desempeñó un rol importante. El ítem "Defiende sus derechos y expresa su opinión sin agredir a los demás" cargó en afabilidad. En el cuestionario usado (ISDP-2; Schmitt et al., 2007), el ítem original era "Tiene una personalidad asertiva", pero los presentes autores lo cambiaron, debido a las dificultades con el término asertivo. Ledesma et al. (2011) comentan que aunque asertivo es en efecto la traducción literal correcta, en su país, Argentina, es un término poco usado en el lenguaje común, ya que su uso se limita a los psicólogos, razón por la cual celebran la explicación que trae Benet-Martínez y John (1998) en su versión: "Es asertivo, no teme expresar lo que quiere". Sin embargo, creímos que esta versión sería problemática, ya que en un país como el nuestro, con una cultura con síndrome de simpatía (Triandis, Marin, Lisansky \& Betancourt, 1984), no está en general bien visto ser afirmativo y seguro, ya que se puede percibir como insolente o descarado. Sin embargo, en la versión que se usó la parte de "sin agredir a los demás", puede estar haciéndolo cargar en afabilidad.

El ítem "es generalmente confiado" cargó débilmente en neuroticismo y afabilidad. Aunque se conserva la misma traducción que en Benet-Martínez y John (1998), el ítem es problemático por dos razones. Primero, la traducción al español trae una acepción negativa. Por otro lado, la falta de confianza general en otros en Colombia (World Value Survey, 2010-2014) puede generar una respuesta sesgada en el contexto colombiano.

Los problemas enfrentados apuntan a la dificultad de la traducción en un cuestionario basado en adjetivos descriptivos de la personalidad. Aunque estos cuestionarios se hayan usado en diferentes países de Latinoamérica, es importante tener en cuenta para su uso los problemas que se identificaron con cinco de los ítems y que se resumen en la tabla 3. En este orden de ideas, se recomienda usar en tres de los casos las versiones originales de Benet-Martínez y John (1998) y cambiar los otros dos ítems a versiones más claras, ya que, como lo mencionamos en los resultados, las palabras asertivo y confiado tienen en nuestro país acepciones que no son positivas. Teniendo en cuenta la recomendación de Schwarz (2007), en estudios a futuro conviene llevar a cabo una prueba cognitiva de los ítems que se prestan a interpretaciones distintas.

Por último, se reprodujeron las diferencias por sexo encontradas por Schmitt et al. (2008) y Bleidorn et al. (2013). Sin embargo, la diferencia solo fue significativa para la dimensión de apertura a la experiencia. Este resultado puede estar relacionado con las características de la muestra. Esta estuvo conformada por jóvenes entre los 17 y los 25 años de edad, con un promedio de 2014, la cual correspondería a lo que Arnett (2000) denomina periodo de la adultez emergente. Aunque la muestra colombiana está exactamente en el rango de edad de los 18 a los 25 años y son estudiantes que están explorando y definiendo su identidad, el nivel socioeconómico medioalto y alto al que pertenecen implica que es poco probable que se hayan emancipado, tengan necesidad de trabajar o hayan definido su rol familiar como madres o padres. Dadas las dinámicas que caracterizan el entorno de estos jóvenes, ellos tienden a ser menos autónomos que sus pares de países desarrollados (Fondo de Población de las Naciones Unidas, 2005).

De lo anterior se colige que algunos de estos jóvenes pueden estar enfrentando los desafíos de la adolescencia avanzada, época en la que según Soto et al. (2008) se observan cambios pronunciados en la personalidad; en ocasiones, en direcciones contrarias a las de la maduración en la edad adulta. Asimismo, si bien se vislumbran algunas diferencias entre mujeres y hombres, estas tienden a ser mínimas. Este resultado, por lo tanto, es un llamado a futuras investigaciones dirigidas a examinar 
la interacción entre sexo y edad en relación con la personalidad en diferentes ámbitos culturales.

Más allá de su contribución, este artículo también tiene limitaciones. Primero, utilizamos una muestra de estudiantes, por lo cual no se pueden generalizar los resultados. Tampoco son representativos de los jóvenes colombianos, por el nivel socioeconómico al que pertenecen y la ubicación de la universidad en la capital del país. Sin embargo, el hecho de que se haya podido copiar la estructura factorial propuesta apoya el uso del cuestionario en muchos campos de la psicología para los cuales esta edad resulta interesante. Segundo, es un trabajo de corte transversal que no presenta otras variables de relación con la personalidad a excepción de la edad y el sexo. La replicación exitosa del BFI en el contexto colombiano abre el camino a estudios que relacionan la personalidad con otras variables como fue mencionado en la introducción.

\section{Referencias}

Allport, G. W. (1937). Personality: A psychological interpretation. New York: Holt.

Arnett, J. J. (2000). Emerging adulthood: A theory of development from the late teens through the twenties. American Psychologist, 55(5), 469-480. Recuperado de http://jeffreyarnett. com/articles/ARNETT_Emerging_Adulthood_ theory.pdf

Bandura, A. (2001). Social cognitive theory: An agentic perspective. Annual Review of Psychology, 52, 1-26. doi: 10.1146/annurev.psych.52.1.1

Barenbaum, N. B. \& Winter, D. G. (2008). History of modern personality theory and research. En O. P. John, R. W. Robins, \& L. A. Pervin (Eds.), Handbook of personality: Theory and research (3a ed., pp. 3-27). New York, NY: Guilford Press. Recuperado de http://es.scribd. com/doc/44970637/Handbook-of-Personality-Third-Edition

Barrick, M. R. \& Mount, M. K. (1991). The Big Five personality dimensions and job performance: $\mathrm{A}$ meta-analysis. Personnel Psychology, 44, 1-26. doi: 10.1111/j.1744-6570.1991.tb00688.x

Bartlett, M. S. (1951). A further note on tests of significance in factor analysis. Bristish Journal of Statistical Psychology, 4(1), 1-2. doi: 10.1111/ j.2044-8317.1951.tb00299.x

Batey, M., Chamorro-Premuzic, T., \& Furnham, A. (2010). Individual differences in ideational behavior. Can the big five and psychometric intelligence predict creativity scores? Creativity Research Journal, 22(1), 90-97. eScholarID:1b5335. doi: 10.1080/10400410903579627

Benet-Martínez, V. \& John, O. P. (1998). Los Cinco Grandes across cultures and ethnic groups: Multitrait multimethod analyses of the Big Five in Spanish and English. Journal of Personality and Social Psychology, 75(3), 729-750. http:// dx.doi.org/10.1037/0022-3514.75.3.729

Berry, J. W. (1980). Introduction to methodology. En H. Triandis \& J. W. Berry (Eds.), Handbook of cross-cultural psychology (vol. 2, pp. 1-28). Boston: Allyn \& Bacon.

Bleidorn, W., Klimstra, T. A., Denissen, J. J. A., Rentfrow, P. J., Potter, J., \& Gosling, S. (2013). Personality maturation around the world: A cross-cultural examination of social-investment theory. Psychological Science, 24(12), 25302540. doi: $10.1177 / 0956797613498396$

Bogg, T. \& Roberts, B. W. (2004). Conscientiousness and health related behaviors: A meta-analysis of the leading behavioral contributors to mortality. Psychological Bulletin, 130(6), 887-919.

Buss, D. M. \& Kenrick, D. T. (1998). Evolutionary social psychology. En D. T. Gilbert, S. T. Fiske, \& G. Lindzey (Eds.), The handbook of social psychology (4th ed.,vol. 2, pp. 982-1026). New York: McGraw-Hill.

Castro-Solano, A. y Casullo, M. M. (2001). Rasgos de personalidad, bienestar psicológico y rendimiento académico en adolescentes argentinos [Personality traits, psychological well-being and academic achievement in Argentine adolescents]. Interdisciplinaria, 18(1), 65-85. Re- 
cuperado de http://www.scielo.org.ar/scielo. php?script $=$ sci_arttext\&lng=pt\&nrm=iso\&tlng=pt\&pid=S1668-70272012000100007

Cattell, R. B. (1943). The description of personality: Principles and findings in a factor analysis. American Journal of Psychology, 58, 69-90.

Christiansen, N. D. \& Tett, R. P. (Eds.) (2013). Handbook of personality at work. New York, NY: Routledge.

Church, A. T. \& Burke, P. J. (1994). Exploratory and confirmatory tests of the Big Five and Tellegen's three and four-dimensional models. Journal of Personality and Social Psychology, 66, 93-114.

Clarke, S. \& Robertson, I. T. (2005). A meta-analytic review of the Big Five personality factors and accident involvement in occupational and non-occupational settings. Journal of Occupational and Organizational Psychology, 78(3), 355-376. doi: 10.1348/096317905X26183

Costa, P. T., Jr. \& McCrae, R. R., (1985). The NEO Personality Inventory manual. Odessa, FL: Psychological Assessment Resources.

Costa, P. T., Jr. \& McCrae, R. R., (1992). Revised NEO Personality Inventory (NEO-PI-R) and NEO Five-Factor Inventory (NEO-FFI) professional manual. Odessa, FL: Psychological Assessment Resources.

Costa, P. T., Jr., Terracciano, A., \& McCrae, R. R., (2001). Gender differences in personality traits across cultures: Robust and surprising findings. Journal of Personality and Social Psychology, 81, 322-331. Recuperado de http://www.cin. ufpe.br/ ssj/Genderdifferences\%20in\%20personality $\% 20$ traits $\% 20$ across $\% 20$ cultures $\% 20$ Robust\%20andsurprising\%20findings.pdf

DeYoung, C. G. \& Gray, J. R. (2009). Personality neuroscience: Explaining individual differences in affect, behavior, and cognition. En P. J. Corr \& G. Matthews (Eds.), The Cambridge handbook of personality psychology (pp. 323-346). New York: Cambridge University Press. Recuperado de http://www.tc.umn.edu/ cdeyoung/
Pubs/DeYoung_2009_personality_neuroscience_Chapter.pdf

Eagly, A. H. (1987). Sex differences in social behavior: A social-role interpretation. Hillsdale, NJ: Erlbaum. http://dx.doi.org/10.1037/0003-066X.52.12.1380.b

Feingold, A. (1994). Gender differences in personality: A meta-analysis. Psychological Bulletin, 116(3), 429-456. http://psycnet.apa.org/index. cfm?fa=buy.optionToBuy\&id=1995-09434-001

Feist, G. J. (1998). A meta-analysis of personality in scientific and artistic creativity. Personality and Social Psychology Review, 2(4), 290-309. Recuperado de http://www.ncbi.nlm.nih.gov/ pubmed/15647135

Fondo de Población de las Naciones Unidas. (2005). Estado de la población mundial 2005: la promesa de igualdad. Equidad de género, salud reproductiva y Objetivos de Desarrollo del Milenio. New York: autor. Recuperado de http:// www.unfpa.org/swp/2005/pdf/sp_swp05.pdf

Foti, R. J. \& Hauenstein, M. A. (2007). Pattern and variable approaches in leadership emergence and effectiveness. Journal of Applied Psychology, 92(2), 347-355. Recuperado de http:// psycnet.apa.org/index.cfm?fa=buy.optionToBuy\&id=1995-09434-001

Gerber, A. S., Huber, G. A., Doherty, D., \& M. Dowling, C. M. (2011). Personality traits and the consumption of political information. American Politics Research, 39(1), 32-84. doi: 10.1177/1532673X10381466

Ghiselli, E. E., Campbell, J. P., \& Zedeck, S. (1981). Measurement theory for the behavioral sciences. San Francisco: Jossey Bass.

Goldberg, L. R. (1981). Language and individual differences: The search for universals in personality lexicons. En L. Wheeler (Ed.), Review of personality and social psychology (vol. 2, pp. 141-165). Beverly Hills: Sage. Recuperado de http://projects.ori.org/lrg/PDFs_papers/universals.lexicon.81.pdf 
Goldberg, L. R. (1992). The development of markers for the Big-Five factor structure. Psychological Assessment, 4, 26-42. http://dx.doi. org/10.1037/1040-3590.4.1.26

Hair, J., Anderson, R. E., Tatham, R., \& Black, W. C. (1995) Multivariate data analysis. (4th Ed.). Upper Saddle River, NJ: Prentice Ha11. Recuperado de http://dl.acm.org/citation. cfm?id=207590

John, O. P., Donahue, E. M., \& Kentle, R. L. (1991). The "Big Five" Inventory-versions $4 a$ and 54. Berkeley: University of California, Berkeley, Institute of Personality and Social Research. Recuperado de http://www.ocf.berkeley.edu/ johnlab/bfi.htm

John, O. P., Naumann, L. P., \& Soto, C. J. (2008a). Paradigm shift to the integrative Big Five trait taxonomy: History, measurement, and conceptual issues. En O. P. John, R. W. Robins, \& L. A. Pervin (Eds.), Handbook of personality: theory and research (3rd ed., pp. 114-158). New York, NY: Guilford Press. Recuperado de http:// es.scribd.com/doc/44970637/Handbook-of-Personality-Third-Edition

John, O. P., Robins, R. W., \& Pervin. L. A. (Eds.). (2008b). Preface. En O. P. John, R. W. Robins, \& L. A. Pervin (Eds.), Handbook of Personality: Theory and Research (3rd ed., pp. xi-xii). New York, NY: Guilford Press. Recuperado de http:// es.scribd.com/doc/44970637/Handbook-of-Personality-Third-Edition

John, O. P. \& Srivastava, S. (2001). The Big Five trait taxonomy: History, measurement, and theoretical perspectives. En L. A. Pervin \& O. P. John (Eds.), Handbook of personality: theory and research (2nd ed., pp. 102-138). New York: Guilford Press.

Judge, T. A., Heller, D., \& Mount, M. K. (2002). Five-Factor Model of personality and job satisfaction: A meta-analysis. Journal of Applied Psychology, 87(3), 530-541. Recuperado de http://www.apa.org/pubs/databases/psycarticles/sample.aspx
Kaiser, H. F. (1970). A second generation Little Jiffy. Psychometrika, 35(4), 401-415. Recuperado de http://link.springer.com/article/10.1007\%2FBF02291817\#page-1

Ledesma, R. D., Sánchez, R., \& Díaz-Lázaro, C. M. (2011). Adjective checklist to assess the Big Five personality factors in the Argentine population. Journal of Personality Assessment, 93(1), 46-55. Recuperado de http://www.unboundmedicine.com/medline/citation/21184330/Adjective_checklist_to_assess_the_big_five_personality_factors_in_the_Argentine_population

Lodi-Smith, J. \& Roberts, B. W. (2007). Social investment and personality: A meta-analysis of the relationship of personality traits to investment in work, family, religion, and volunteerism. Personality and Social Psychology Review, 11, 68-86. doi:10.1177/1088868306294590

Lorenzo-Seva, U. \& ten Berge, J. M. F. (2006). Tucker's congruence coefficient as a meaningful index of factor similarity. Methodology: European Journal of Research Methods for the Behavioral and Social Sciences, 2(2), 57-64. http://dx.doi. org/10.1027/1614-2241.2.2.57

Markus, H. R. \& Kitayama, S. (1991). Culture and the self: Implications for cognition, emotion, and motivation. Psychological Review, 98(2), 224-253. Recuperado de http://psycnet.apa.org/index.cfm?fa=buy.option ToBuy\&uid=1991-23978-001

McCrae, R. R. \& Costa, Jr., P. T. (1992). An introduction to the five factor model and its applications. Journal of Personality, 60, 175-215.

McCrae, R. R., Costa, Jr., P. T., Hrebícková, M., Urbánek, T., Martin, T. A., Oryol, V. E., Rukavishnikov, A. A., \& Senin, I. G. (2004). Age differences in personality traits across cultures: Self-report and observer perspectives. $E u$ ropean Journal of Personality, 18, 143-157. doi:10.1002/per.510

McCrae, R. R. et al. (2005). Universal features of personality traits from the observer's perspective: Data from 50 cultures. Journal of Personality 
and Social Psychology, 88, 547-561. Recuperado de http://jlporrata.net/pdf/ppoc1.pdf

Millon, T. (2012). On the history and future study of personality and its disorders. Annual Review of Clinical Psychology, 8, 1-19. Recuperado de http://www.unboundmedicine.com/medline/ citation/22035244/On_the_history_and_future_study_of_personality_and_its_disorders

Mischel, W. \& Shoda, Y. (1995). A cognitive-affective system theory of personality: Reconceptualizing situations, dispositions, dynamics, and invariance in personality structure. Psychological Review, 102, 246-268. Recuperado de http://www. homepage.psy.utexas.edu/HomePage/Class/ Psy394U/Bower/11\%20Soc\%20Cog\%20Personality/Mischel-Shoda.pdf

Murray, H. (1938). Explorations in personality. New York: Oxford University Press. Recuperado de https://archive.org/stream/explorationsinpe031973mbp/explorationsinpe031973mbp_djvu.txt

Nisbett, R. E. (2003). The geography of thought: How Asians and Westerners think differently... And why. New York, NY: Free Press.

Ortiz, F. A., Church, A. T., Vargas-Flores, J. de J., Ibáñez-Reyes, J., Flores-Galaz, M., Iuit-Briceño, J. I., \& Escamilla, J. M. (2007). Are indigenous personality dimensions culture-specific? Mexican inventories and the Five-Factor Model. Journal of Research in Personality, 41, 618-649.

Poropat, A. E. (2009). A meta-analysis of the five-factor model of personality and academic performance. Psychological Bulletin, 135(2), 322-38. doi: 10.1037/a0014996

Real Academia Española. (2001). Diccionario de la lengua española (22. ${ }^{\mathrm{a}}$ ed.). Recuperado de http://www.rae.es/drae/

Roberts, B. W., Wood, D., \& Smith, J. L. (2005). Evaluating five factor theory and social investments perspectives on personality trait development. Journal of Research in Personality, 39, 166-184. Recuperado de http:// www.google.com.co/url?sa=t\&rct=j\&q=\&es- $\mathrm{rc}=\mathrm{s} \&$ source $=$ web $\& \mathrm{~cd}=4 \& \mathrm{ved}=0 \mathrm{CCwQFjA}-$ D\&url=http $\% 3 \mathrm{~A} \% 2 \mathrm{~F} \% 2 \mathrm{Fwww}$.researchgate. net $\% 2$ Fprofile\%2FBrent_Roberts\%2Fpublication\%2F221953546_Evaluating_Five_Factor_ Theory_and_social_investment_perspectives_ on_personality_trait_development\%2Flinks\%2F0046353c23bf98d210000000\&ei=SgZlVLCBOIuXNvDPgfAJ\&usg=AFQjCNHK_c-clqYP9RuFBcZwJVMyw9Y2Ww\&sig2=W2L4CAi0GvgQUayrbn4EGw

Ryan, R. M. \& Deci, E. L. (2000). Self-determination theory and the facilitation of intrinsic motivation, social development, and well-being. American Psychologist, 55, 68-78. Recuperado de http://www.ncbi.nlm.nih.gov/pub$\mathrm{med} / 11392867$

Schmitt, D. P., Alcalay, L., Allensworth, M., Allik, J., Ault, L., Austers, I., et al. (2002). Patterns and universals of adult romantic attachment across 62 cultural regions: Are models of self and others pancultural constructs? Journal of Cross-Cultural Psychology, 35(4), 367-402.

Recuperado de http://www.researchgate.net/ publication/223956426_Patterns_and_universals_of_adult_romantic_attachment_ across_62_cultural_regions_Are_models_of Self_and_Other_pancultural_constructs

Schmitt, D. P., Allik, J., McCrae, R. R., \& Benet-Martínez, V., et al. (2007). The geographic distribution of Big Five personality traits: Patterns and profiles of human self-description across 56 nations. Journal of Cross-Cultural Psychology, 38, 173-212. Recuperado de http://www. bradley.edu/dotAsset/165904.pdf

Schmitt, D. P., Realo, A., Voracek, M., \& Allik, J. (2008). Why can't a man be more like a women?: Sex differences in Big Five personality traits across 55 cultures. Journal of Personality and Social Psychology, 94, 168-182. Recuperado de http://www.academia.edu/6162205/ Why_Cant_a_Man_Be_More_Like_a_Woman_Sex_Differences_in_Big_Five_Personality_Traits_Across_55_Cultures 
Schwarz, N. (2007). Cognitive aspects of survey methodology. Applied Cognitive Psychology, 21, 277-287. Recuperado de http://deepblue.lib.umich.edu/bitstream/handle/2027.42/55956/1340_ftp.pdf

Soto, C. J., John, O. P., Gosling, S. D., \& Potter, J. (2008). The developmental psychometrics of Big Five self-reports: Acquiescence, factor structure, coherence, and differentiation from ages 10 to 20. Journal of Personality and Social Psychology, 94(4), 718-737. http://dx.doi. org/10.1037/0022-3514.94.4.718

Soto, C. J., John, O. P., Gosling, S. D., \& Potter, J. (2010). Age difference in personality traits from 10 to 65: Big Five domains and facets in a large cross-sectional sample. Journal of Personality and Social Psychology, 100(2), 330348. Recuperado de http://www.researchgate. net/publication/49696299_Age_differences in_personality_traits_from_10_to_65_Big_Five_domains_and_facets_in_a large_cross-sectional_sample

Soubelet, A. \& Salthouse, T. A. (2011). Influence of social desirability on age differences in self-reports of mood and personality. Journal of Personality, 79, 741-762. doi: 10.1111/j.14676494.2011.00700.x

Srivastava, S., John, O. P., Gosling, S. D., \& Potter, J. (2003). Development of personality in early and middle adulthood: Set like plaster or persistent change? Journal of Personality and Social
Psychology, 84(5), 1041-1053. Recuperado de http://pages.uoregon.edu/sanjay/pubs/b5development.pdf

Triandis, H., Marin, G., Lisansky, J., \& Betancourt, H. (1984). Simpatia as a cultural script of Hispanics. Journal of Personality and Social Psychology, 47, 1363-1374.

Tucker, L. R. (1951). A method for synthesis of factor analysis studies (Personnel Research Section Report 984). Washington, DC: Department of the Amy.

Tupes, E.C. \& Christal, R. E. (1961). Recurrent personality factors based on trait ratings. (Technical Report ASD-TR-61-97). Lackland Air Force Base, TX: Personnel Laboratory, Air Force Systems Command.

Vigil-Colet, A., Morales-Vives, F., \& Lorenzo-Seva, U. (2013). How social desirability and acquiescence affect the age-personality relationship. Psicothema, 25(3), 342-346.

Widiger, T. A. \& Mullins-Sweatt, S. N. (2009). Five-factor model of personality disorders. Psychological Assessment, 17, 278-287.

World Values Survey Wave 6 2010-2014 Official Aggregate v.20140429. World Values Survey Association (www.worldvaluessurvey.org). Aggregate File Producer: Asep/JDS, Madrid SPAIN.

Zuckerman, M. (1991). Psychobiology of personality. Cambridge: Cambridge University Press. 